\title{
A Novel Synthetic Route of Fused Tricyclic Framework Quinoline Derivatives from Readily Available Aliphatic Amino Carboxylic Acid Substrates
}

\author{
SHIREEN MOHAMMED \\ Department of Chemistry, Faculty of Science, University of Zakho, KIR-IRAQ, Iraq. \\ ${ }^{*}$ Corresponding author E-mail: shireen2222@yahoo.com \\ http://dx.doi.org/10.13005/ojc/350215
}

(Received: November 21, 2018; Accepted: March 14, 2019)

\begin{abstract}
A novel and an efficient strategy of fused tricyclic quinoline heterocycle compounds from aliphatic amino carboxylic acid substrates was studied. The protocol here is proceed over main reaction processes including: cyclization, protection, amidine formation, further cyclization and finally coupling with boronic acid substrate through Suzuki reaction. These reactions afforded the corresponding products in high yields. Furthermore, all synthesized compounds were identified by spectral data.
\end{abstract}

Keywords: Aliphatic amino carboxylic acid, Protection reactions, Cyclization, Diazotization, nosylation reactions, Suzuki reaction, Cross-coupling process, Palladium catalyst.

\section{INTRODUCTION}

The heterocyclic compounds have a major interest role in medicinal chemistry ${ }^{1}$, mild molecules furnishing with variety substituted functional groups consider to get much attention, due to their potential to design multiple chemical library. Meanwhile, nitrogen inclusive heterocycles such as quinolines propose specific advantage because of their different coordination of biological activities. For example, quinoline compounds were reported to control broad spectrum of beneficial biological activities universal anti-tuberculosis ${ }^{2}$, antiproliferative ${ }^{3}$ anthelmintic ${ }^{4}$, antibacterial ${ }^{5}$, antioxidant activities $^{6}$ antimalarial ${ }^{7}$, inhibitors of oncogenic Ras $^{8}$, antitumor ${ }^{9}$, antimicrobial ${ }^{10}$ anti-neoplastic ${ }^{11}$, anti-oxidant ${ }^{12}$ activity and antiviral $^{13}$. In 1834, the first extraction of quinoline from coal tar was reported by Friedlieb Ferdinand Runge ${ }^{14}$.

Hence, because of these reasons plentiful attentiveness is paid for the synthesis and the biological value of quinoline nucleus, our current work is an amplification of our outstanding efforts toward design and developed novel and facile protocol towered synthesis of new fused tricyclic quinoline derivatives from readily available aliphatic amino carboxylic acid. Stable tricyclic heterocycle product compounds yields were obtained over

This is an Open Access article licensed under a Creative Commons license: Attribution 4.0 International (CC- BY). Published by Oriental Scientific Publishing Company @ 2018

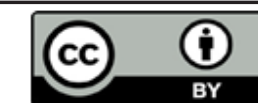


several reaction processes, started with cyclization ${ }^{15}$ and protection of aliphatic amino carboxylic acid substrates, and followed by amidine formation ${ }^{16}$, further cyclization ${ }^{17}$, diazotization ${ }^{18}$, nosylation ${ }^{19}$ and finally coupling with boronic acid substrate through Suzuki reaction process ${ }^{20}$ (Scheme 1).

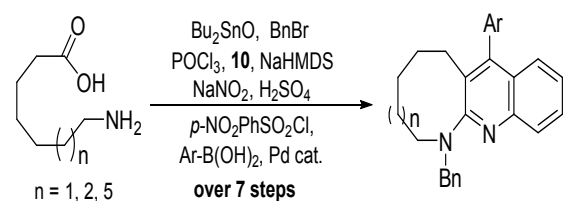

Scheme 1. Synthetic route of fused tricyclic framwork quinoline subtituents

\section{RESULT AND DISCUSSION}

In these precursory studies, we concentrated our effort on the construction of the fused tricyclic quinoline moiety starting from the readily available aliphatic amino carboxylic acid substrates (1-3). The planning (Scheme 2 ) of these reactions started with an effective the amide bond formation through an intramolecular cyclization reaction in the presence of $\mathrm{Bu}_{2} \mathrm{SnO}$ under refluxing toluene, using a Dean-Stark apparatus ${ }^{21}$. While, a simple procedure for protection of amino groups (4-6) with benzyl group was applied under basic conditions to give the expected products (7-9) with high yields. Subsequently, the coupling of protected lactams (4-6) with 2-(trifluoromethyl)aniline 10 in presence of $\mathrm{POCl}_{3}$ reagent ${ }^{22}$ via Friedlander reaction should allow an access to an amidine intermediate (11-13). Furthermore, cyclization of the latter produced an aromatic amine (14-16), which under diazotization reaction conditions afforded the corresponding phenol products (17-19) in high yields. Conversion the phenol to the corresponding nosylate compounds (21-23), through reaction with p-nitrosulfonyl chloride 20 , was reported to be a quite functional process. Finally, palladiumcatalyzed cross-coupling ${ }^{23}$ of nosylate compounds (2123) with aryl boronic acid substrate 24 and employing Suzuki reaction, provided the required products (25-27) with very good yields.

Generally, the proposed mechanism (Scheme 3) of preparation of lactams in the presence of $\mathrm{Bu}_{2} \mathrm{SnO}$ under refluxing toluene proceeds initial with stannylation of aliphatic amino carboxylic acid substrates (1-3) at the carboxylic acid group (1-3)i. This awards the way to the formation of a hydroxystannylene amino carboxylate intermediate (1-3)ii, which can undergo intramolecular cyclization to produce the desired lactam products (4-6) with removal of water molecule.

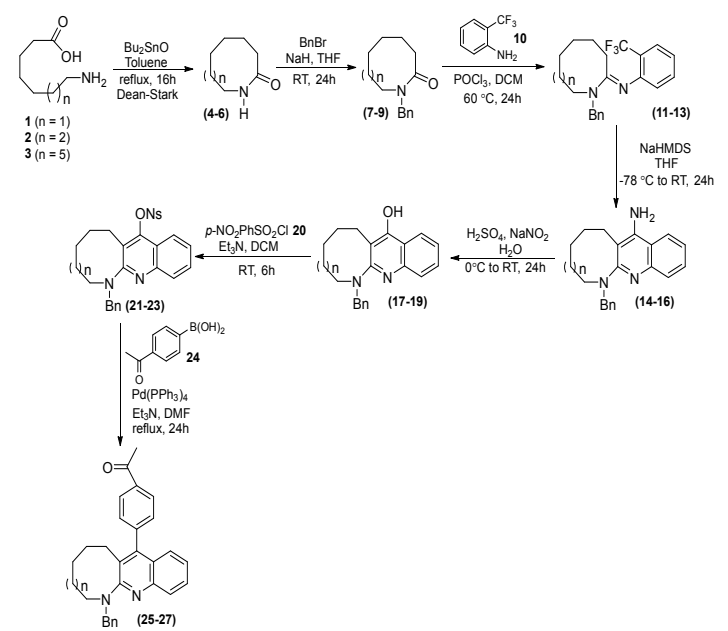

Scheme 2. Synthesis of tricyclic quinolones form aliphatic amino carboxylic acid substrates

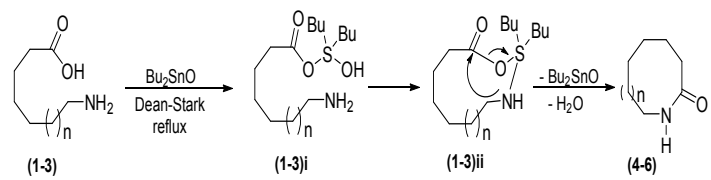

Scheme 3. Proposed mechanism of intermolecular cyclization

Therefore, the suggested mechanism for the qualified cyclization of amidines is outlined in (Scheme 4). The first proton abstraction from (11-13) compounds in presence of base, lead to form the stable anion intermediate at $-78^{\circ} \mathrm{C}$. While increasing the temperature, it undergoes elimination of fluoride anion and give the quinone methide intermediate species. Further generation of anion in intermediates with the base, eliminate the second equivalent of fluoride. Finally, the resultant can undergo aromatization to afford the expected products (14-16) with the losing HF molecule ${ }^{17}$.

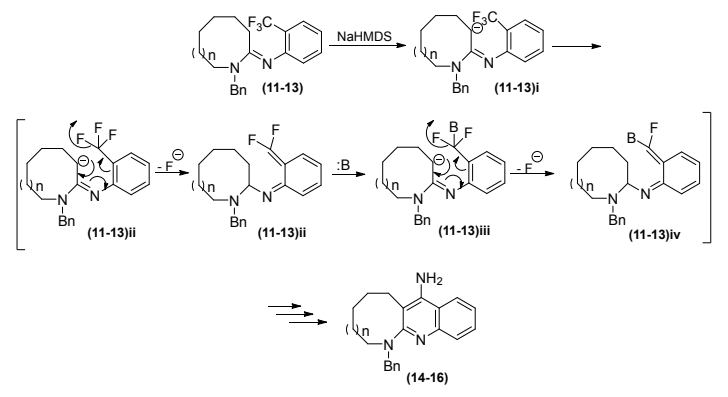

Scheme 4. Proposed mechanism of intermolecular imidine intermediates cyclization 


\section{EXPERIMENTAL}

Melting points were registered uncorrected ${ }^{1} \mathrm{H}$ - and ${ }^{13} \mathrm{C}$-NMR spectra were obtained with Bruker DPX-300 FT-NMR spectrometer. The NMR spectra sitting in this article were measured in deuterium chloroform solution. IR spectra were reported with a Perkin-EImer 1710-FTIR spectrometer. Revelation was carried out UV light at 254-365 $\mathrm{nm}$. The purification was done through the flash chromatography process using Merck Silica Gel.

General protocol for synthesis of Lactams: An amino acids (0.63 mmol, 1 eq.) and di-nbutyloxide ( $156.8 \mathrm{mg}, 0.63 \mathrm{mmol}, 1$ eq.) were stirred in refluxing toluene $(120 \mathrm{~mL})$ for $16 \mathrm{~h}$ with use of a Dean-Stark system for the continuous distilling out the water. The solvent evaporated in vacuo at room temperature and the dissolved in $\mathrm{CHCl}_{3}(25 \mathrm{~mL})$ and passed via short layer of Celite. The resultant was concentrated under vacuum and purified through the chromatography to produce the products (4-6) as a white solid.

Azocan-2-one (4): (81\%). (83-85) ${ }^{\circ} \mathrm{C}$. IR $v_{\max }\left(\mathrm{cm}^{-1}\right)=3320,1685,1645,1465,1331,790 .{ }^{1} \mathrm{H}$ NMR: $\delta(p p m)=6.10(\mathrm{~s}, 1 \mathrm{H}), 3.54$ (apppearant t, $2 \mathrm{H}, \mathrm{J}=3$ and $\left.6 \mathrm{~Hz}, \mathrm{CH}_{2}\right), 2.93\left(\mathrm{t}, 2 \mathrm{H}, \mathrm{J}=3 \mathrm{~Hz}, \mathrm{CH}_{2}\right)$, 2.02-1.96 (m, 4H, $\left.2 \mathrm{CH}_{2}\right), 1.74-1.66\left(\mathrm{~m}, 4 \mathrm{H}, 2 \mathrm{CH}_{2}\right)$. ${ }^{13} \mathrm{C}$ NMR: $\delta(\mathrm{ppm})=175.9(\mathrm{C}=\mathrm{O}), 41.7(\mathrm{C}), 37.3(\mathrm{C})$, $29.1(\mathrm{C}), 28.7(\mathrm{C}), 27.2(\mathrm{C}), 24.9(\mathrm{C})$.

Azonan-2-one (5): (79\%). (103-105) ${ }^{\circ} \mathrm{C}$. IR $v_{\max }\left(\mathrm{cm}^{-1}\right)=3325,1681,1540,1330,795 .{ }^{1} \mathrm{H}$ NMR: $\delta(\mathrm{ppm})=5.91(\mathrm{~s}, 1 \mathrm{H}), 3.30\left(\mathrm{t}, 2 \mathrm{H}, J=3 \mathrm{~Hz}, \mathrm{CH}_{2}\right)$, 2.84 (t, $\left.2 \mathrm{H}, J=3 \mathrm{~Hz}, \mathrm{CH}_{2}\right), 1.91-1.84\left(\mathrm{~m}, 4 \mathrm{H}, 2 \mathrm{CH}_{2}\right)$, 1.61-1.56 (m, $\left.6 \mathrm{H}, 3 \mathrm{CH}_{2}\right) \cdot{ }^{13} \mathrm{C}$ NMR: $\delta(\mathrm{ppm})=175.9$ $(\mathrm{C}=\mathrm{O}), 41.1(\mathrm{C}), 37.7(\mathrm{C}), 30.0(\mathrm{C}), 29.5(\mathrm{C}), 29.5$ (C), $28.0(\mathrm{C}), 25.7(\mathrm{C})$.

Azacyclododecan-2-one (6): (77\%). (119$121)^{\circ} \mathrm{C}$. IR $v_{\text {max }}\left(\mathrm{cm}^{-1}\right)=3324,1686,1645,1466$, 1332, 790. ${ }^{1} \mathrm{H}$ NMR: $\delta(\mathrm{ppm})=5.98(\mathrm{~s}, 1 \mathrm{H}), 3.25$ (t, $2 \mathrm{H}, J=3 \mathrm{~Hz}, \mathrm{CH}_{2}$ ), 2.44 (appearant t, $2 \mathrm{H}, J=3$ and $\left.6 \mathrm{~Hz}, \mathrm{CH}_{2}\right), 1.87-1.81\left(\mathrm{~m}, 4 \mathrm{H}, 2 \mathrm{CH}_{2}\right), 1.58-1.52$ $\left(\mathrm{m}, 12 \mathrm{H}, 6 \mathrm{CH}_{2}\right) \cdot{ }^{13} \mathrm{C}$ NMR: $\delta(\mathrm{ppm})=175.9(\mathrm{C}=\mathrm{O})$, $41.1(\mathrm{C}), 37.8(\mathrm{C}), 30.0(\mathrm{C}), 29.5(3 \mathrm{C}), 29.5(2 \mathrm{C})$, $28.0(\mathrm{C}), 25.7(\mathrm{C})$.

General protocol for synthesis of Benzyl Lactams: A solution of $\mathrm{NaH}(6.8 \mathrm{mg}, 0.312 \mathrm{mmol}$,
1.5 eq.) in dry $\operatorname{THF}(10 \mathrm{~mL})$ at $0^{\circ} \mathrm{C}$, lactams (4-6) (0.205 mmol, 1 eq.) were added with stirring for 1 hour. The ice removed and keep stirring for $24 \mathrm{~h}$ at room temperature. The solvent removed under vacuum, followed by purification using flash chromatography to yield the products (7-9) as a pale yellow solid.

1-benzylazocan-2-one (7): (84\%). (105$107)^{\circ} \mathrm{C} . \mathrm{IR} v_{\max }\left(\mathrm{cm}^{-1}\right)=3070,1950,1810,1666$, 1451, 770. ${ }^{1} \mathrm{H}$ NMR: $\delta(\mathrm{ppm})=7.33-7.21(\mathrm{~m}, 5 \mathrm{H}$, CHar), 4.49 (s, 2H, $\mathrm{CH}_{2}$ ), $3.52\left(\mathrm{t}, 2 \mathrm{H}, \mathrm{J}=3 \mathrm{~Hz}, \mathrm{CH}_{2}\right.$ ), $2.86\left(\mathrm{t}, 2 \mathrm{H}, \mathrm{J}=6 \mathrm{~Hz}, \mathrm{CH}_{2}\right), 1.91-1.86\left(\mathrm{~m}, 2 \mathrm{H}, \mathrm{CH}_{2}\right)$, 1.82-1.78 (m, 2H, $\left.\mathrm{CH}_{2}\right), 1.61-1.57\left(\mathrm{~m}, 2 \mathrm{H}, \mathrm{CH}_{2}\right)$, 1.54-1.49 (m, 2H, $\left.\mathrm{CH}_{2}\right) .{ }^{13} \mathrm{C}$ NMR: $\delta(\mathrm{ppm})=176.5$, 138.0, 128.8 (2), 128.6 (2), 127.7, 49.6, 47.2, 37.4 , 28.2, 27.0, 26.8, 24.8 .

1-benzylazonan-2-one (8): (87\%). (119$121)^{\circ} \mathrm{C} . \mathrm{IR} v_{\max }\left(\mathrm{cm}^{-1}\right)=3071,1952,1814,1665$, 1451, 770. ${ }^{1} \mathrm{H}$ NMR: $\delta(\mathrm{ppm})=7.38-7.20(\mathrm{~m}, 5 \mathrm{H}$, CHar), 4.51 (s, 2H, $\mathrm{CH}_{2}$ ), 3.42 (t, $2 \mathrm{H}, \mathrm{J}=3 \mathrm{~Hz}, \mathrm{CH}_{2}$ ), 2.41 (appearant t, $2 \mathrm{H}, \mathrm{J}=3$ and $6 \mathrm{~Hz}, \mathrm{CH}_{2}$ ), 1.83-1.77 $\left(\mathrm{m}, 4 \mathrm{H}, 2 \mathrm{CH}_{2}\right), 1.55-1.49\left(\mathrm{~m}, 6 \mathrm{H}, 3 \mathrm{CH}_{2}\right) .{ }^{13} \mathrm{C}$ NMR: $\delta(\mathrm{ppm})=175.4,137.9,128.7$ (2), 128.5 (2), 127.5, 49.4, 46.4, 36.8, 29.0, 29.0, 27.7, 27.0, 25.5.

1-benzylazacyclododecan-2-one (9): (79\%). (123-125) ${ }^{\circ} \mathrm{C}$. IR $v_{\max }\left(\mathrm{cm}^{-1}\right)=3070,1951$, 1811, 1664, 1450, 770. ${ }^{1} \mathrm{H}$ NMR: $\delta(\mathrm{ppm})=7.31-7.19$ (m, 5H, CHar), $4.48\left(\mathrm{~s}, 2 \mathrm{H}, \mathrm{CH}_{2}\right), 3.43-4.36(\mathrm{~m}, 2 \mathrm{H}$, $\mathrm{CH}_{2}$ ), 2.38 (appearant $\mathrm{t}, 2 \mathrm{H}, \mathrm{J}=3$ and $6 \mathrm{~Hz}, \mathrm{CH}_{2}$ ), 1.81-1.73 (m, 4H, $\left.2 \mathrm{CH}_{2}\right), 1.52-1.46\left(\mathrm{~m}, 12 \mathrm{H}, 6 \mathrm{CH}_{2}\right)$. ${ }^{13} \mathrm{C}$ NMR: $\delta(\mathrm{ppm})=175.6,138.1,128.9(2), 128.7$ (2), 127.7, 49.9, 46.6, 37.0, 29.2 (3), 29.2 (2), 27.9, $27.8,25.7$.

General protocol for synthesis of Imidines: To benzyl lactams (7-9) (3.61 mmol, 1 eq.) in freshly distillated DCM (10 mL), $\mathrm{POCl}_{3}(0.66 \mathrm{~mL}, 7.2 \mathrm{mmol}$, 2 eq.) was added quietly. 2 -(trifluoromethyl)aniline 10 (0.61 gm, 3.7 mmol, 1.05 eq.) in DCM was added quietly. The mixture was heating under reflux at $60^{\circ} \mathrm{C}$ for 24 hours. Ice-cold water was added followed by a $25 \% \mathrm{NaOH}$ solution to $\mathrm{pH} 8$. The extraction was done with $\mathrm{DCM}$, brine, drying over $\mathrm{MgSO}_{4}$, the organic solvents were concentrated under vacuum and the resultant was purified by chromatography to yield the desired products (11-13) as yellow oil. 
1-benzyl-N-(2-(trifluoromethyl)phenyl) azocan-2-imine (11): ): (78\%). IR $v_{\max }\left(\mathrm{cm}^{-1}\right)=3071$, 2942, 2217, 1580, 1377, 1237, 1088, 1205, 1020, 750, 730. ${ }^{1} \mathrm{H}$ NMR: $\delta(\mathrm{ppm})=7.64-7.63(\mathrm{~m}, 1 \mathrm{H}$, CHar), 7.43-7.24 (m, 8H, $\left.\mathrm{CH}_{2}\right), 4.68\left(\mathrm{~s}, 2 \mathrm{H}, \mathrm{CH}_{2}\right)$, $3.71(\mathrm{t}, 1 \mathrm{H}, J=3 \mathrm{~Hz}, \mathrm{CHa}), 3.40(\mathrm{t}, 1 \mathrm{H}, J=3 \mathrm{~Hz}$, $\mathrm{CHb}), 2.81\left(\mathrm{t}, 2 \mathrm{H}, \mathrm{J}=3 \mathrm{~Hz}, \mathrm{CH}_{2}\right), 1.90-1.86(\mathrm{~m}, 2 \mathrm{H}$, $\left.\mathrm{CH}_{2}\right), 1.81-1.76\left(\mathrm{~m}, 2 \mathrm{H}, \mathrm{CH}_{2}\right), 1.63-1.58(\mathrm{~m}, 4 \mathrm{H}$, $\left.2 \mathrm{CH}_{2}\right) \cdot{ }^{13} \mathrm{C}$ NMR: $\delta(\mathrm{ppm})=145.2,138.5,134.3$, 129.1 (2), 129.0 (2), 128.7, 128.1, 125.9, 125.2, $124.6,123.1,121.4,52.0,50.7,32.6,28.7,27.3$, $27.2,26.4$.

1-benzyl-N-(2-(trifluoromethyl)phenyl) azonan-2-imine (12): (81\%). IR $v_{\max }\left(\mathrm{cm}^{-1}\right)=3072$, 2941, 2219, 1584, 1378, 1237, 1088, 1206, 1020, 750, 730. ${ }^{~} \mathrm{H}$ NMR: $\delta(\mathrm{ppm})=7.65(\mathrm{~d}, 1 \mathrm{H}, \mathrm{J}=6 \mathrm{~Hz}$, CHar), 7.43-7.26 (m, 8H, CHar), 4.08 (s, 2H, $\mathrm{CH}_{2}$ ), $3.61(\mathrm{t}, 1 \mathrm{H}, J=3 \mathrm{~Hz}, \mathrm{CHa}$ ), 3.52 (appearant t, $2 \mathrm{H}$, $J=3$ and $6 \mathrm{~Hz}, \mathrm{CHb}$ ), 2.52 (t, $2 \mathrm{H}, J=3 \mathrm{~Hz}, \mathrm{CH}_{2}$ ), 1.86-1.75 (m, $\left.4 \mathrm{H}, 2 \mathrm{CH}_{2}\right), 1.96-1.49\left(\mathrm{~m}, 6 \mathrm{H}, 3 \mathrm{CH}_{2}\right)$. ${ }^{13} \mathrm{C}$ NMR: $\delta(\mathrm{ppm})=176.4,144.6,138.1,133.8$, 128.6 (2), 128.4 (2), 127.6, 125.4, 124.7, 124.1, 122.6, 121.1, 51.5, 49.6, 33.3, 29.1, 29.0, 27.7, 27.6, 26.9.

1-benzyl-N-(2-(trifluoromethyl)phenyl) azacyclododecan-2-imine (13): (77\%). IR $v_{\text {max }}\left(\mathrm{cm}^{-1}\right)$ $=3070,2941,2217,1580,1378,1237,1089,1204$, 1020, 751, 730. ${ }^{1} \mathrm{H}$ NMR: $\delta(\mathrm{ppm})=7.58(\mathrm{~d}, 1 \mathrm{H}, J=$ $6 \mathrm{~Hz}, \mathrm{CHar}$ ), 7.36-7.19 (m, 8H, CHar), 4.62 (s, 2H, $\left.\mathrm{CH}_{2}\right)$, 3.55-3.51 (m, $\left.2 \mathrm{H}, \mathrm{CH}_{2}\right), 2.46(\mathrm{t}, 2 \mathrm{H}, J=3 \mathrm{~Hz}$, $\mathrm{CHb}), 1.80-1.69\left(\mathrm{~m}, 4 \mathrm{H}, 2 \mathrm{CH}_{2}\right), 1.50-1.43(\mathrm{~m}, 12 \mathrm{H}$, $\left.6 \mathrm{CH}_{2}\right) \cdot{ }^{13} \mathrm{C}$ NMR: $\delta(\mathrm{ppm})=167.7,144.9,138.2$, 134.0, 129.0 (2), 128.5 (2), 127.8, 125.6, 124.2, 122.9, 121.4, 121.1, 51.8, 49.9, 33.6, 29.4 (3), 29.3 (2), 28.1, 27.9, 27.2.

General protocol for the Cyclization: To the imidines (11-13) (5.1 mmol, 1 eq.) in stirring dry THF were added to a vigorously solution of Sodium bis(trimethylsilyl)amide solution NaHMDS (4 molar eq.) at $-78{ }^{\circ} \mathrm{C}$ for 20 minutes. The reaction warmed slowly to $-20^{\circ} \mathrm{C}$ for 1 hour, then at room temperature for 24 hours. The workup proceeded through quenched with brine then extraction with EtOAc $(3 X 100 \mathrm{~mL})$. The resultant transact over $\mathrm{Na}_{2} \mathrm{SO}_{4}$ then concentrated under vacuum. The purification via the flash chromatography, afforded the expected products (14-16) as red solid. 1-benzyl-1,2,3,4,5,6-hexahydroazocino [2,3-b]quinolin-7-amine (14): (83\%). (134-136) ${ }^{\circ} \mathrm{C}$. IR $v_{\max }\left(\mathrm{cm}^{-1}\right)=3184,2931,1670,1571,1501,1448$, 1376, 1072, 1019, 735. ${ }^{1} \mathrm{H}$ NMR: $\delta(\mathrm{ppm})=8.07$ (d, $1 \mathrm{H}, J=3 \mathrm{~Hz}, \mathrm{CHar}$ ), 7.71 (dd, $1 \mathrm{H}, J=3$ and 6 $\mathrm{Hz}$, CHar), 7.65-7.48 (m, 6H, CHar), 7.40-7.37 (m, $1 \mathrm{H}, \mathrm{CHar}), 4.90\left(\mathrm{~s}, 2 \mathrm{H}, \mathrm{CH}_{2}\right), 4.10(\mathrm{t}, 1 \mathrm{H}, \mathrm{J}=3 \mathrm{~Hz}$, $\mathrm{CHa}), 3.81\left(\mathrm{~s}, 2 \mathrm{H}, \mathrm{CH}_{2}\right), 3.62(\mathrm{t}, 1 \mathrm{H}, J=3 \mathrm{~Hz}, \mathrm{CHb})$, $2.69\left(\mathrm{t}, 2 \mathrm{H}, \mathrm{J}=3 \mathrm{~Hz}, \mathrm{CH}_{2}\right), 2.04-2.00\left(\mathrm{~m}, 2 \mathrm{H}, \mathrm{CH}_{2}\right)$, 1.94-1.89 (m, 2H, $\left.\mathrm{CH}_{2}\right), 1.70-1.66\left(\mathrm{~m}, 2 \mathrm{H}, \mathrm{CH}_{2}\right)$. ${ }^{13} \mathrm{C}$ NMR: $\delta(\mathrm{ppm})=163.0,153.5,141.9,137.9,130.2$, 130.0, 128.7 (2), 128.5 (2), 127.6, 124.6, 123.2, 115.5, 114.4, 52.9, 49.6, 29.0, 26.6, 26.2, 25.6.

1-benzyl-2,3, 4, 5, 6, 7-hexahydro-1Hazonino[2,3-b]quinolin-8-amine (15): (87\%). (154156) $)^{\circ} \mathrm{C} . \mathrm{IR} v \max \left(\mathrm{cm}^{-1}\right)=3175,2931,1672,1570$, 1501, 1448, 1376, 1072, 1019, 735. ${ }^{1} \mathrm{H}$ NMR: $\delta$ (ppm) $=7.88(\mathrm{dd}, 1 \mathrm{H}, J=3$ and $6 \mathrm{~Hz}, \mathrm{CHar}), 7.51$ (dd, $1 \mathrm{H}$, $J=3$ and $6 \mathrm{~Hz}, \mathrm{CHar}), 7.46-7.30(\mathrm{~m}, 6 \mathrm{H}, \mathrm{CHar})$, 7.20-7.17 (m, $1 \mathrm{H}, \mathrm{CHar}), 4.71\left(\mathrm{~s}, 2 \mathrm{H}, \mathrm{CH}_{2}\right), 3.95$ (s, $\left.2 \mathrm{H}, \mathrm{CH}_{2}\right), 3.63-3.58\left(\mathrm{~m}, 2 \mathrm{H}, \mathrm{CH}_{2}\right), 2.58(\mathrm{t}, 2 \mathrm{H}$, $\left.\mathrm{J}=3 \mathrm{~Hz}, \mathrm{CH}_{2}\right), 1.96-1.91\left(\mathrm{~m}, 2 \mathrm{H}, \mathrm{CH}_{2}\right), 1.86-1.80(\mathrm{~m}$, $\left.2 \mathrm{H}, \mathrm{CH}_{2}\right), 1.61-1.55\left(\mathrm{~m}, 4 \mathrm{H}, \mathrm{CH}_{2}\right) \cdot{ }^{13} \mathrm{C}$ NMR: $\delta$ (ppm) $=166.1,153.7,142.1,138.0,130.3,130.0,128.8(2)$, 128.6 (2), 127.6, 124.8, 123.3, 115.7, 114.4, 53.1, $44.2,30.0,29.0,27.7,27.7,25.8$.

1-benzyl-1,2,3,4,5,6,7,8,9,10-decahydro[1]azacyclododecino[2,3-b]quinolin-11-amine (16): (85\%). $(177-179)^{\circ} \mathrm{C} . \mathrm{IR} v_{\max }\left(\mathrm{cm}^{-1}\right)=3178,2931$, 1670, 1571, 1509, 1450, 1376, 1072, 1020, 735. ${ }^{1} \mathrm{H}$ NMR: $\delta(\mathrm{ppm})=7.79(\mathrm{~d}, 1 \mathrm{H}, J=6 \mathrm{~Hz}, \mathrm{CHar}), 7.46$ (d, $1 \mathrm{H}, J=3 \mathrm{~Hz}, \mathrm{CHar}), 7.32-7.21(\mathrm{~m}, 6 \mathrm{H}, \mathrm{CHar})$, 7.10-7.07 (m, $1 \mathrm{H}, \mathrm{CHar}), 4.61\left(\mathrm{~s}, 2 \mathrm{H}, \mathrm{CH}_{2}\right), 3.67$ (s, $2 \mathrm{H}, \mathrm{CH}_{2}$ ), 3.58 (appearant t, $1 \mathrm{H}, \mathrm{J}=3$ and $6 \mathrm{~Hz}$, $\mathrm{CHa}$ ), 3.49 (t, $1 \mathrm{H}, \mathrm{J}=3 \mathrm{~Hz}, \mathrm{CHb}$ ), 2.49 (appearant $\mathrm{t}, 2 \mathrm{H}, \mathrm{J}=3$ and $\left.6 \mathrm{~Hz}, \mathrm{CH}_{2}\right), 1.66-1.61\left(\mathrm{~m}, 2 \mathrm{H}, \mathrm{CH}_{2}\right)$, 1.76-1.73 (m, 2H, $\left.\mathrm{CH}_{2}\right), 1.53-1.46\left(\mathrm{~m}, 10 \mathrm{H}, \mathrm{CH}_{2}\right)$. ${ }^{13} \mathrm{C}$ NMR: $\delta(\mathrm{ppm})=166.1,156.1,153.8,142.0$, 138.0, 130.3, 130.0, 128.8 (2), 128.6 (2), 127.6, 124.8, 123.3, 53.0, 49.0, 30.0, 29.2 (3), 29.0 (2), $27.7,27.6,25.8$.

General protocol for Preparation of Phenols: To the amines (14-16) (0.2 mmol, 1 eq.) and $\mathrm{H}_{2} \mathrm{SO}_{4}$ $\left(53 \mu \mathrm{mL}, 1 \mathrm{mmol}, 5\right.$ eq.), $\mathrm{NaNO}_{2}(690 \mathrm{mg}, 1 \mathrm{mmol}$, 5 eq.) in $\mathrm{H}_{2} \mathrm{O}(2.5 \mathrm{~mL})$ was added dropwise at $0^{\circ} \mathrm{C}$, and then stirring $24 \mathrm{~h}$ at room temperature. A $25 \%$ $\mathrm{NaOH}$ solution was added quietly at $0^{\circ} \mathrm{C}$ to $\mathrm{pH}=8-9$. The product was extracted with EtOAc, brine, dried 
over $\mathrm{MgSO}_{4}$, then evaporation the solvent in vacuo. The purification through flash chromatography, gave the expected products (17-19) as a white solid.

1-benzyl-1,2,3,4,5,6-hexahydroazocino [2,3-b]quinolin-7-ol (17): (70\%). (203-205) ${ }^{\circ} \mathrm{C}$. IR $v_{\max }\left(\mathrm{cm}^{-1}\right)=3340,2975,1620,1386,1499,1473$, 1090, 1050, 885, 742. ${ }^{1} \mathrm{H}$ NMR: $\delta(\mathrm{ppm})=7.82(\mathrm{dd}$, $1 \mathrm{H}, J=3$ and $6 \mathrm{~Hz}, \mathrm{CHar}), 7.71$ (dd, $1 \mathrm{H}, J=3$ and $6 \mathrm{~Hz}, \mathrm{CHar}$ ), 7.37-7.15 (m, 7H, CHar), 4.62 (s, 2H, $\mathrm{CH}_{2}$ ), 3.62 (t, $1 \mathrm{H}, \mathrm{J}=3 \mathrm{~Hz}, \mathrm{CHa}$ ), 3.34 (appearant $\mathrm{t}, 1 \mathrm{H}, J=3$ and $6 \mathrm{~Hz}, \mathrm{CHb}), 2.45(\mathrm{t}, 2 \mathrm{H}, J=3 \mathrm{~Hz}$, $\left.\mathrm{CH}_{2}\right)$, 1.75-1.72 (m, 2H, $\left.\mathrm{CH}_{2}\right), 1.66-1.61(\mathrm{~m}, 2 \mathrm{H}$, $\left.\mathrm{CH}_{2}\right), 1.42-1.37\left(\mathrm{~m}, 3 \mathrm{H}, \mathrm{CH}_{2}\right) .{ }^{13} \mathrm{C}$ NMR: $\delta(\mathrm{ppm})=$ 169.1, 163.8, 142.7, 138.2, 131.4, 129.0 (2), 128.8, $127.8,127.9,127.3,125.2,124.3,122.6,115.2$, 53.3, 50.0, 29.3, 27.1, 26.1, 24.0.

1-benzyl-2,3,4,5,6,7-hexahydro-1Hazonino[2,3-b]quinolin-8-ol (18): (74\%). (215-217 C. IR $v_{\max }\left(\mathrm{cm}^{-1}\right)=3342,2975,1621,1389,1450,1473$, 1090, 1050, 885, 742. ${ }^{1} \mathrm{H}$ NMR: $\delta(p p m)=7.81(\mathrm{dd}$, $1 \mathrm{H}, J=3$ and $6 \mathrm{~Hz}, \mathrm{CHar}), 7.62$ (dd, $1 \mathrm{H}, J=3$ and $9 \mathrm{~Hz}, \mathrm{CHar}$ ), 7.35-7.14 (m, 7H, CHar), 4.61 (s, 2H, $\left.\mathrm{CH}_{2}\right)$, 3.52-3.49 (m, 2H, $\left.\mathrm{CH}_{2}\right), 2.53(\mathrm{t}, 2 \mathrm{H}, \mathrm{J}=3 \mathrm{~Hz}$, $\mathrm{CHb})$, 1.87-1.83 (m, 2H, $\left.\mathrm{CH}_{2}\right), 1.76-1.71(\mathrm{~m}, 2 \mathrm{H}$, $\left.\mathrm{CH}_{2}\right), 1.52-1.46\left(\mathrm{~m}, 4 \mathrm{H}, 2 \mathrm{CH}_{2}\right), 1.40(\mathrm{~s}, 1 \mathrm{H}, \mathrm{OH})$. ${ }^{13} \mathrm{C}$ NMR: $\delta(\mathrm{ppm})=169.0,167.1,142.7,138.3$, 131.6, 129.1 (2), 129.0 (2), 128.1, 127.5, 125.3, $124.5,122.7,115.5,53.3,49.4,30.3,29.2,28.1$, 28.1, 23.7.

1-benzyl-1,2,3,4,5,6,7,8,9,10-decahydro[1] azacyclododecino[2,3-b]quinolin-11-ol (19): (73\%). (235-237) $)^{\circ} \mathrm{C} . \quad I R v_{\max }\left(\mathrm{cm}^{-1}\right)=3340,2975$, 1620, 1386, 1499, 1479, 1095, 1047, 885, 742. ${ }^{1} \mathrm{H}$ NMR: $\delta(\mathrm{ppm})=7.86(\mathrm{~d}, 1 \mathrm{H}, J=6 \mathrm{~Hz}, \mathrm{CHar})$, 7.66 (s, 1H, CHar), 7.28-7.14 (m, 7H, CHar), 4.58 (s, $\left.2 \mathrm{H}, \mathrm{CH}_{2}\right), 3.51-3.46\left(\mathrm{~m}, 2 \mathrm{H}, \mathrm{CH}_{2}\right), 2.51$ (appearant t, $2 \mathrm{H}, \mathrm{J}=3$ and $\left.6 \mathrm{~Hz}, \mathrm{CH}_{2}\right), 1.86-1.79\left(\mathrm{~m}, 2 \mathrm{H}, \mathrm{CH}_{2}\right)$, 1.74-1.69 (m, 2H, $\left.\mathrm{CH}_{2}\right), 1.51-1.44\left(\mathrm{~m}, 10 \mathrm{H}, 5 \mathrm{CH}_{2}\right)$. ${ }^{13} \mathrm{C}$ NMR: $\delta(\mathrm{ppm})=168.7,166.3,142.2,137.8,128.6$ (2), 128.3 (2), 127.5, 124.7, 123.9, 122.3, 115.5, 52.9, 49.9, 29.7, 29.1, 28.8 (4), 27.5 (3), 23.2.

General Procedure of Nosylations: To a solution of phenols (17-19) (0.2 mmol, 1 eq.) in freshly distillated DCM $(2.2 \mathrm{~mL})$, was added triethyl amine (20 $\mu \mathrm{mL}, 0.2 \mathrm{mmol}, 1$ eq.) with stirring 30 $\min$ at $0^{\circ} \mathrm{C}$. Then $\mathrm{p}$-nitrobenzene sulfonyl chloride 20 (44 mg, 0.2 mmol, 1 eq.) in DCM was added quietly to the mixture of the reaction mixture then stirring for 6 hours at room temperature. The resultant was concentrated and dissolved in EtOAc, followed by washing with $1 \mathrm{~N} \mathrm{HCl}, 1 \mathrm{~N} \mathrm{NaOH}$, saturated $\mathrm{NaCl}$ solution. The organic layer was removed by vacuum. The purifiecation through flash chromatography, produced the expected products (21-23) as yellow solid

1-benzyl-1,2,3,4,5,6-hexahydroazocino [2,3-b]quinolin-7-yl 4-nitrobenzenesulfonate (21): (79\%). (182-184) $)^{\circ} \mathrm{C}$. IR (ATR) $v_{\max }\left(\mathrm{cm}^{-1}\right)=2962$, 1615, 1258, 1084, 1014, 791, 745. ${ }^{1} \mathrm{H}$ NMR: $\delta$ (ppm) $=7.46(\mathrm{~d}, 2 \mathrm{H}, J=6 \mathrm{~Hz}, \mathrm{CHar}), 8.29(\mathrm{~d}, 2 \mathrm{H}, J=6$ $\mathrm{Hz}, \mathrm{CHar}$ ), 7.77 (dd, $1 \mathrm{H}, \mathrm{J}=3$ and $6 \mathrm{~Hz}, \mathrm{CHar}), 7.75$ (d, $1 \mathrm{H}, J=6 \mathrm{~Hz}, \mathrm{CHar}), 7.29-7.16$ (m, 7H, CHar), $4.61\left(\mathrm{~s}, 2 \mathrm{H}, \mathrm{CH}_{2}\right), 3.62(\mathrm{t}, 1 \mathrm{H}, \mathrm{J}=3 \mathrm{~Hz}, \mathrm{CHa}), 3.33$ (t, $1 \mathrm{H}, J=3 \mathrm{~Hz}, \mathrm{CHb}), 2.46\left(\mathrm{t}, 2 \mathrm{H}, J=3 \mathrm{~Hz}, \mathrm{CH}_{2}\right.$ ), 1.75-1.71 (m, 2H, $\left.\mathrm{CH}_{2}\right), 1.60-1.55\left(\mathrm{~m}, 2 \mathrm{H}, \mathrm{CH}_{2}\right)$, 1.40-1.35 (m, 2H, $\left.\mathrm{CH}_{2}\right) .{ }^{13} \mathrm{C}$ NMR: $\delta(p p m)=164.9$, 159.0, 147.5, 146.1, 145.0, 138.1, 132.1, 131.2, 130.6 (2), 129.0, $128.8(2), 128.7$ (2), $127.6(2), 124.5,124.2$ (2), 123.6, 53.0, 49.7, 29.2, 26.8, 25.8, 24.1.

1-benzyl-2,3,4,5,6,7-hexahydro-1 Hazonino[2,3-b]quinolin-8-yl 4-nitrobenzenesulfonate (22): (86\%). (197-199) oC. IR (ATR) $v_{\max }\left(\mathrm{cm}^{-1}\right)$ $=2961,1617,1259,1085,1015,794,744 .{ }^{1} \mathrm{H}_{\mathrm{N}} \mathrm{NR}$ : $\delta(\mathrm{ppm})=8.50(\mathrm{~d}, 2 \mathrm{H}, \mathrm{J}=3 \mathrm{~Hz}, \mathrm{CHar}), 8.24(\mathrm{~d}, 2 \mathrm{H}$, $J=6 \mathrm{~Hz}, \mathrm{CHar}), 7.85$ (d, 1H, $J=3 \mathrm{~Hz}, \mathrm{CHar}), 7.57$ (d, $1 \mathrm{H}, J=6 \mathrm{~Hz}, \mathrm{CHar})$, 7.32-7.30 (m, 6H, CHar), 7.15-7.12 (m, 1H, CHar), $4.62\left(\mathrm{~s}, 2 \mathrm{H}, \mathrm{CH}_{2}\right), 3.56-3.51$ $\left(\mathrm{m}, 2 \mathrm{H}, \mathrm{CH}_{2}\right), 2.57\left(\mathrm{t}, 2 \mathrm{H}, \mathrm{J}=3 \mathrm{~Hz}, \mathrm{CH}_{2}\right), 1.88-1.84$ $\left(\mathrm{m}, 2 \mathrm{H}, \mathrm{CH}_{2}\right), 1.77-1.72\left(\mathrm{~m}, 2 \mathrm{H}, \mathrm{CH}_{2}\right), 1.53-1.48$ $\left(\mathrm{m}, 4 \mathrm{H}, 2 \mathrm{CH}_{2}\right) \cdot{ }^{13} \mathrm{C}$ NMR: $\delta(\mathrm{ppm})=167.9,159.0$, $147.5,145.1,138.0,132.1,131.3,130.6$ (2), 129.1, 128.8 (2), 128.6 (2), 128.0, 127.8 (2), 124.5, 124.2, 123.5, 53.1, 49.2, 30.1, 29.1, 27.8 (2), 27.7, 23.7.

1-benzyl-1,2,3,4,5,6,7,8,9,10-decahydro[1] azacyclododecino[2,3-b]quinolin-11-yl 4-nitrobenzenesulfonate (23): $(89 \%)$. $(210-212)^{\circ} \mathrm{C}$. IR $($ ATR $) v_{\max }\left(\mathrm{cm}^{-1}\right)=2959,1619,1259,1084,1012$, 794, 735. ${ }^{1} \mathrm{H}$ NMR: $\delta(\mathrm{ppm})=8.49(\mathrm{~d}, 2 \mathrm{H}, J=6 \mathrm{~Hz}$, CHar), 8.23 (d, 2H, J=6 Hz, CHar), 7.85 (dd, 2H, $J=3$ and $6 \mathrm{~Hz}, \mathrm{CHar}), 7.30-7.19(\mathrm{~m}, 7 \mathrm{H}, \mathrm{CHar}), 4.61$ (s, $2 \mathrm{H}, \mathrm{CH}_{2}$ ), 3.53-3.50 (m, 2H, $\left.\mathrm{CH}_{2}\right), 2.58$ (appearant $\mathrm{t}, 2 \mathrm{H}, \mathrm{J}=3$ and $\left.9 \mathrm{~Hz}, \mathrm{CH}_{2}\right), 1.87-1.82\left(\mathrm{~m}, 2 \mathrm{H}, \mathrm{CH}_{2}\right)$, 1.77-1.71 (m, 2H, $\left.\mathrm{CH}_{2}\right), 1.52-1.46\left(\mathrm{~m}, 10 \mathrm{H}, 3 \mathrm{CH}_{2}\right)$. ${ }^{13} \mathrm{C}$ NMR: $\delta(\mathrm{ppm})=167.9,159.1,147.5,146.1$, 145.2, 138.1, 132.0, 131.4 (2), 130.6, 129.1, 128.9 (2), 128.7 (2), 128.1, 127.7, 124.2 (2), 123.5, 53.1, 49.2, 30.1, 29.2 (3), 29.0 (2), 27.7, 27.7, 23.6. 
General Procedure for the Coupling Reaction: To the Nosylate compounds (21-23) (0.18 $\mathrm{mmol}, 1$ eq.) in DMF ( $5 \mathrm{~mL})$, Boronic acid substrate 24 (0.56 mmol, 2 eq.) and $\mathrm{Et}_{3} \mathrm{~N}(30 \mathrm{~mL}, 0.2 \mathrm{mmol}$, 1.2 eq.) were added. The solution was bubbled for 30 minutes, then Pd-catalyst $(21 \mathrm{mg}, 0.018 \mathrm{mmol}$, 0.1 eq.) was added and reflux overnight. The solvent evaporated under vacuum. The purification of the crude mixture through chromatography, gave the pure products (25-27) as a white solid.

1-(4-(1-benzyl-1,2,3,4,5,6-hexahydroazocino [2,3-b]quinolin-7-yl)phenyl)ethan-1-one (25): $(89 \%) .(239-241)^{\circ} \mathrm{C}$. IR (ATR) $v_{\max }\left(\mathrm{cm}^{-1}\right)=$ $3075,2924,1710,1560,1493,1431,1395$, 1366, 1346, 1170, 741. ${ }^{1} \mathrm{H}$ NMR: $\delta(\mathrm{ppm})=8.01$ (d, 2H, $J=6 \mathrm{~Hz}, \mathrm{CHar}), 7.93$ (d, $1 \mathrm{H}, J=3 \mathrm{~Hz}, \mathrm{CHar}$ ), $7.73(\mathrm{~d}, 2 \mathrm{H}, J=3 \mathrm{~Hz}, \mathrm{CHar}), 7.39$ (d, $1 \mathrm{H}, J=3 \mathrm{~Hz}$, CHar), 7.31-7.27 (m, 4H, CHar), 7.25-7.19 (m, 2H, CHar), 7.12-7.09 (m, 1H, CHar), $4.62\left(\mathrm{~s}, 2 \mathrm{H}, \mathrm{CH}_{2}\right)$, $3.82(\mathrm{t}, 1 \mathrm{H}, J=3 \mathrm{~Hz}, \mathrm{CHa}$ ), 3.34 (appearant t $1 \mathrm{H}, J$ $=3$ and $6 \mathrm{~Hz}, \mathrm{CHb}), 2.99\left(\mathrm{~s}, 3 \mathrm{H}, \mathrm{CH}_{3}\right), 2.44(\mathrm{t}, 2 \mathrm{H}, \mathrm{J}$ $\left.=3 \mathrm{~Hz}, \mathrm{CH}_{2}\right), 1.75-1.59\left(\mathrm{~m}, 2 \mathrm{H}, \mathrm{CH}_{2}\right), 1.63-1.59(\mathrm{~m}$, $\left.2 \mathrm{H}, \mathrm{CH}_{2}\right), 1.41-1.36\left(\mathrm{~m}, 2 \mathrm{H}, \mathrm{CH}_{2}\right) \cdot{ }^{13} \mathrm{C}$ NMR: $\delta(\mathrm{ppm})$ $=197.7,163.0,153.1,144.5,138.8,137.9,137.0$, $135.7,131.5,130.1$ (2), 129.1, 128.5 (2), 128.3, 128.1, 127.8 (2), 127.5 (2), 125.7, 124.3, 52.8, 49.5, 36.0, 29.0, 27.7, 26.6, 25.5 .

1-(4-(1-benzyl-2,3,4,5,6,7-hexahydro-1Hazonino[2,3-b]quinolin-8-yl)phenyl)ethan-1-one (26): (85\%). (230-232) ${ }^{\circ} \mathrm{C}$. IR (ATR) vmax $\left(\mathrm{cm}^{-1}\right)$ $=3074,2924,1711,1560,1490,1429,1390,1366$, $1346,1170,742 .{ }^{1} \mathrm{H}$ NMR: $\delta(\mathrm{ppm})=8.01(\mathrm{~d}, 2 \mathrm{H}, J$ $=9 \mathrm{~Hz}, \mathrm{CHar}), 7.93$ (dd, $1 \mathrm{H}, J=3$ and $6 \mathrm{~Hz}, \mathrm{CHar}$ ), $7.73(\mathrm{~d}, 2 \mathrm{H}, J=3 \mathrm{~Hz}, \mathrm{CHar}), 7.40$ (d, $1 \mathrm{H}, J=6 \mathrm{~Hz}$, CHar), 7.32-7.11 (m, 7H, CHar), $4.62\left(\mathrm{~s}, 2 \mathrm{H}, \mathrm{CH}_{2}\right)$, 3.56-3.51 (m, $\left.2 \mathrm{H}, \mathrm{CH}_{2}\right), 2.99\left(\mathrm{~s}, 3 \mathrm{H}, \mathrm{CH}_{3}\right), 2.53$ (appearant $\mathrm{t}, 2 \mathrm{H}, \mathrm{J}=6$ and $9 \mathrm{~Hz}, \mathrm{CH}_{2}$ ), 1.88-1.83 $\left(\mathrm{m}, 2 \mathrm{H}, \mathrm{CH}_{2}\right), 1.77-1.72\left(\mathrm{~m}, 2 \mathrm{H}, \mathrm{CH}_{2}\right), 1.53-1.48(\mathrm{~m}$, $\left.4 \mathrm{H}, 2 \mathrm{CH}_{2}\right) .{ }^{13} \mathrm{C}$ NMR: $\delta(\mathrm{ppm})=199.1,167.8,155.0$, 146.3, 140.7, 139.7, 138.6, 137.6, 133.5, 131.8,
$130.9,130.5,130.2$ (2), 129.8, 129.8, 129.4, 127.5 (2), 126.2 (2), 54.7, 50.7, 36.5, 31.7, 30.7, 30.6, 29.6, 29.4, 29.3.

1-(4-(1-benzyl-1,2,3,4,5,6,7,8,9,10decahydro-[1]azacyclododecino[2,3-b]quinolin-11yl)phenyl)ethan-1-one (27): (81\%). (251-253) ${ }^{\circ} \mathrm{C}$. IR (ATR) $v_{\max }\left(\mathrm{cm}^{-1}\right)=3075,2925,1711,1560,1493$, 1431, 1395, 1370, 1346, 1170, 741. ${ }^{1} \mathrm{H}$ NMR: $\delta$ (ppm) $=7.99(\mathrm{~d}, 2 \mathrm{H}, J=3 \mathrm{~Hz}, \mathrm{CHar}), 7.96(\mathrm{dd}, 1 \mathrm{H}, J=3$ and $6 \mathrm{~Hz}, \mathrm{CHar}), 7.70(\mathrm{~d}, 2 \mathrm{H}, J=3 \mathrm{~Hz}, \mathrm{CHar}), 7.39$ (d, $1 \mathrm{H}, J=3 \mathrm{~Hz}, \mathrm{CHar}), 7.29-7.11(\mathrm{~m}, 7 \mathrm{H}, \mathrm{CHar})$, $4.61\left(\mathrm{~s}, 2 \mathrm{H}, \mathrm{CH}_{2}\right.$ ), 3.52 (t, $\left.2 \mathrm{H}, J=3 \mathrm{~Hz}, \mathrm{CH}_{2}\right), 2.57$ (s, 3H, $\mathrm{CH}_{3}$ ), 2.54 (appearant t, $2 \mathrm{H}, J=3$ and $6 \mathrm{~Hz}$, $\left.\mathrm{CH}_{2}\right)$, 1.87-1.82 (m, 2H, $\left.\mathrm{CH}_{2}\right), 1.77-1.72(\mathrm{~m}, 2 \mathrm{H}$, $\left.\mathrm{CH}_{2}\right), 1.52-1.46\left(\mathrm{~m}, 10 \mathrm{H}, 5 \mathrm{CH}_{2}\right) \cdot{ }^{13} \mathrm{C}$ NMR: $\delta(\mathrm{ppm})$ $=199.0,167.8,155.1,140.7,139.7,137.6$ (2), 133.4 (2), 131.9 (2), 130.9, 130.4 (2), 129.8 (2), 129.4 (2), 127.4 (2), 126.1 (2), 54.7, 50.7, 36.6, 31.7, 30.8 (3), 30.6, 29.7, 29.4, 29.3.

\section{CONCLUSION}

In outline, we have presented a functional and useful across the construction of fused tricyclic heterocyclic quinolines. This method was developed and started first with the cyclization and protection of aliphatic amino carboxylic acids and then followed by the amidine formation through the Vlismeier intermediate. Moreover, the cyclization process with 2-(trifluoromethyl)aniline substrate, provided the amino compounds. Transformation of the latter to the corresponding hydroxyl then nosylate analogies, pliable the conclusive Suzuki reaction to proceed simply in the involvement of aromatic boronic acid substrate and affording the expected products in high yields.

\section{ACKNOWLEDGEMENT}

Authors is grateful to the Faculty of Science, Chemistry Department, Zakho University for providing all facilities.

\section{REFERENCES}

1. (a) Cravotto, G., Nano, G.M., Palmisano, G., Tagliapietra, S., Tetrahedron Asymmetry., 2001, 12, 707-709; (b). Kayser, O., Kolodziej, H., Planta Med., 1997, 63, 508-510; (c) Wang, C.J., Hsieh, Y. J., Chu, C.Y., Lin, Y. L., Tseng, T. H., Cancer Lett., 2002, 183, 163-168; (d) Kirkiacharian, S., Thuy, D.T., Sicsic, S.,
Bakhchinian, R., Kurkjian, R., Tonnaire, T., Farmaco., 2002, 57, 703-708.

2. (a) Puneet, P. J., Mariam, S. D, Archana, R., Muktikanta, R., Rajan, M. G., Rational drug design based synthesis of novelarylquinolines as anti-tuberculosis agents, Bioorg. Med. Chem. Lett., 2013, 23, 6097-6105; 
(b) Upadhayaya, R. S., Kulkarni, G. M., Vasireddy, N. R., Vandavasi, J. K., Dixit S. S., Sharma, V. et al. Design, synthesis and biologicalevaluation of novel triazole, urea and thiourea derivatives of quinoline against Mycobacterium tuberculosis, Bioorg. Med. Chem., 2009, 17, 4681-4692

3. (a) Yeh, L. C., Chao, J. H., Zun, Y. H., Chih, H. T., Feng, S. C., Sheng, H. Y. Bioorg. Med. Chem., 2006, 14, 3098-3105; (b) Eun, J. K., Mohammed, I. E., Chang, H. O., So, H. L., Taebo, S., Garam, K. Eur. J. Med. Chem., 2013, 70, 10-21.

4. Sharon, R., Jean, M. P., Whitfield, P. J., Jonesa, K., Bioorg. Med. Chem. Let., 2005, 15, 4806-4808.

5. Kidwai, M., Bhushan, K. R., Sapra, P., Saxena, R. K., Gupta, R., Bioorg. Med. Chem., 2000, 8, 69-72.

6. Nuran, K., Busra, Y., Ayca, A., Zeynep, I., Fatih, S. B., Nurettin, Y., Eur. J. Med. Chem., 2013, 69, 348-355.

7. (a) Curd, F. H.S, Raison, C. G., Rose, F. L,. J. Chem. Soc., 1947, 899-909; (b) Gemma, S., Kukreja, G., Fottorusso, C., Persico, M., Romano, M. P., Altarelli, M. Bioorg. Med. Chem. Lett., 2006, 16, 5384-5388; (c) Mukesh, C. J., Kathryn, J.W., Dale, T., Roger, H., Peter, J. S., Timothy J. E., Eur. J. Med. Chem., 2013, 69, 338-347.

8. (a) El-Sayed, O. A., Aboul-Enein, H. Y., Arch. Pharm. (Weinheim)., 2001, 334, 117-120; (b) Wolin, R., Wang, D., Kelly, J., Afonso, A., James, L., Kirschmeier, P., Mcphail, A. T., Bioorg. Med. Chem. Lett., 1996, 6, 195- 200.

9. Tsuzuki, Y., Yomita, K., Sato, Y., Bioorg. Med. Chem. Lett., 2004, 14, 1216.

10. Antoine, M., Barreau, M., Descon, C., Philippe, G., Guy, P., US Pat., 2003, 65, 48506.

11. Catoen, C., Facompre, M., Houssin, R., Pommery, N., Goossens, J., Colson, P., J. Med. Chen., 2004, 47, 3665-3673.

12. Aoblovich, M., Prenzler, P. D., Pabalides, E., McDonald, S., Robards, K., Analyst., 2002, 127, 183-198.

13. Benard, C., Zoubiri, F., Nor, B. N., Danet, M.,
Desmele, D., Leh, H., Bioorg. Med. Chem. Lett., 2004, 14, 2473- 2476.

14. (a) Chisholm, H., Quinoline. Encyclopædia Britannica, $11^{\text {th }}$ ed., Cambridge University, 759, 1911; (b) Runge, F. F., Annalen der Physik und Chemie., 1834, 31(5), 65-78.

15. Tsui, S.-K.; Wood, J. D. Can. J. Chem., 1979, 57, 1977-1979.

16. (a)Mohammed, S., Robert, F., Landais, Y., Development of new radical processes: approaches toward the synthesis of Eucophylline, thesis., 2014.;(b) Hassan, H., Mohammed, S., Robert, F., Landais, Y., Org. Lett., 2015, 17(18), 4518-4521.;(c) Mohammed, S., Maher, K., Indian J. Hetero. Chem., 2019, 29(1), 21-25.

17. Smith, L., Kiselyov, A. S., Tetrahedron Lett., 1999, 40(31), 5643-5646.

18. (a) Patai, S., Chemistry of the Diazonium and Diazo Groups: Part 1., Ed., WileyBlackwell, 1978; (b) Patai, S., Chemistry of the Diazonium and Diazo Groups: Part 2., Ed., Wiley-Blackwell., 1978.

19. Ciattini, P. G.; Morera, E.; Ortar, G. Tetrahedron Lett., 1992, 33, 4815-4818.

20. (a) Suzuki A., Pure \& Appl. Chem., 1991, 63, 419; (b) Norio, M., Akira, S., Chemical Reviews., 1995, 95, 2457-2483.

21. (a) Dean, E. W., Stark, D., D., The Journal of Industrial \& Engineering Chemistry., 1960, 12(5), 486-490; (b) Wiberg, K., B., Laboratory Technique in Organic Chemistry. McGrawHill series in advanced chemistry. New York: McGraw Hill., 1960.

22. (a) Mohammed, S., Maher, K., Indian J. Hetero. Chem., 2017, 27, 1-6; (b) Mohammed, S., Maher, K., Indian J. Hetero. Chem., 2017, 27, 83-87.; (c) Mohammed, S., Khalid, M., Orient. J. Chem., 2015, 31(4), 2137-2146.

23. (a) Miyaura, N., Yamada, K., Suzuki, A., Tetrahedron Lett. 1979, 20, 3437-3440; (b) Miyaura N. and Suzuki A., Chem. Comm., 1979, 19, 866-867; (c) Miyaura N. and Suzuki A., Chemical Reviews., 1995, 95, 2457-2483. 